\title{
Evaluation of Retrograde Interlocking Intramedullary Sign Nailing in Distal Third Femoral Shaft Fracture
}

\author{
Dr. Erfanul Huq siddiqui ${ }^{*}$, Dr. Md. Sazzad Hossain ${ }^{2}$, Dr. Md Shamsul Alam ${ }^{3}$, Dr. Sheikh Forhad ${ }^{4}$, Dr. Jannat Sultana ${ }^{5}$
}

\begin{abstract}
${ }^{1}$ Consultant, Department of Orthopaedic Surgery, Bangabandhu Sheikh Mujib Medical University (BSMMU), Dhaka, Bangladesh ${ }^{2}$ Professor, Department of Orthopaedic Surgery, Bangabandhu Sheikh Mujib Medical University (BSMMU), Dhaka, Bangladesh ${ }^{3}$ Junior consultant, Department of Orthopaedic Surgery, Gazaria Upazila Health Complex, Munshigonj, Bangladesh

${ }^{4}$ Consultant, Department of Orthopaedic Surgery, Bangabandhu Sheikh Mujib Medical University (BSMMU), Dhaka, Bangladesh ${ }^{5}$ Medical Officer, Department Paediatric Medicine, Bangabandhu Sheikh Mujib Medical University (BSMMU), Dhaka, Bangladesh
\end{abstract}

DOI: $10.36347 /$ sjams.2021.v09i04.019

| Received: 06.03.2021 | Accepted: 27.03.2021 | Published: 19.04.2021

*Corresponding author: Dr. Erfanul Huq Siddiquie

Abstract

Original Research Article

Background: Retrograde intramedullary nailing is a good surgical option in the management of distal femur fracture with osteoporosis. Fractures of the thighbone that occur just above the knee joint are called distal femur fractures. The distal femur is where the bone flares out like an upside-down funnel. The distal femur is the area of the leg just above the knee joint. Methodology: The prospective Study was conducted in the Department of Orthopaedic Surgery of Dhaka Medical College Hospital, Dhaka, Bangladesh to find out the common indications of Distal Third Femoral Shaft Fracture. Clinical examination and evaluation were done from January 2005 to December 2006. Other necessary investigations were done if clinically indicated and to prepare the patient for anesthesia. 15 cases were randomly selected for the study whose common indication of Distal Third Femoral Shaft Fracture. In most cases, injury was high energy trauma due to motor vehicle accident and there was a preponderance of fracture on the right side (70\%) than the left side (30\%). All patients reported after trauma and were resuscitated and fixed at earlier possible date. Maximum patients were operated upon within 2 weeks. Statistical analysis of the results was obtained by using window-based computer software devised with Statistical Packages for Social Sciences (SPSS-22). Results: Mean age of the patients was 40.5 years and majority of the patients $(86.66 \%)$ were male with male-female ratio $6.5: 1$ Six patients developed delayed union. At the end of follow-up, functional scoring was done on the basis of hospital for special surgery score and the results were excellent in $9(30 \%)$ cases, good in $14(46.66 \%)$, fair in $4(13.33 \% 6)$ and poor in $3(10 \%)$ cases. Conclusions: The management of distal femoral shaft fractures constitutes a therapeutic dilemma; the problems are primarily related to basic anatomic structure and biomechanical stresses of this region. Conservative method resulted in high rates of morbidity. So, open reduction and internal fixation is the treatment of choice in distal third femoral fracture.

Keywords: Evaluation; Distal Third Femoral; Shaft Fracture; Interlocking; Intramedullary; Sign; Nail.

Copyright $\odot 2021$ The Author(s): This is an open-access article distributed under the terms of the Creative Commons Attribution 4.0 International License (CC BY-NC 4.0) which permits unrestricted use, distribution, and reproduction in any medium for non-commercial use provided the original author and source are credited.

\section{INTRODUCTION}

The advent of interlocking nails has greatly expanded the indications for intramedullary nailing and successfully controlling the axial and rotational deforming forces, especially in distal third and comminuted fractures. The technique started in early 80 's and quickly popularized all over the world due to its effectiveness in controlling rotational and axial malalignment. Fracture of the shaft of the femur are among the most common fractures encountered in orthopaedic practice and fractures of distal third of the femur are historically difficult to treat. These fractures are often the result of high -energy trauma and may be associated with multiple system injuries. Because of the proximity of the knee-joint to the distal femur, full knee motion and function may be difficult. The problems of malalignment and shortening, especially rotational, are common in lower third shaft fractures. The history of femoral fractures management reflects this underlying dilemma. People tried to manage femoral fractures by splinting or casting up to late nineteenth century [1]. After the introduction of traction pin apparatus in 1907 by Steinmann and in 1909 by Kirschner, traction becomes the most popular technique tor femoral fracture management [2]. The application of Thomas splint and functional cast bracing technique has improved and refined management. However, the basic 
Erfanul Huq siddiqui et al; Sch J App Med Sci, Apr, 2021; 9(4): 583-587

traction technique remained unchanged until now. The management of femoral fractures was revolutionized by the introduction of intramedullary nail by some study [3]. The excellent clinical results resulted in wide dissemination of the techniques, which has been refined over last five decades. But traditional kuntscher nail cannot prevent rotation or angulation even in stable fractures in distal third of femur.

Over the years, many people with many devices tried to resolve this acute problem in distal third fractures. But none gained universal acceptance because of inefficient control of malalignment and technical difficulties and complications.

The antegrade femoral nail allows for better control of proximal shaft fractures, while the retrograde femoral nail is more reliable in controlling distal shaft fractures [4]. Both proximal and distal shafts of the femur have wide medullary cavity, and angulation and rotation of fracture fragments are common in these regions. Wider proximal portion of the nails occupy proximal and distal capacious areas in case of antegrade and retrograde inserted nails respectively and resist angulation and rotation. Recently several retrograde intramedullary nails (SIGN, BROMET, ACE) have been designed to specifically address distal femoral shaft and supracondylar fractures. These interlocking nails differ in design from each other but all are placed in a retrograde fashion. We are doing retrograde "SIGN" (Surgical implant Generation Network) nailing for distal femoral shaft fractures I NITOR (National Institute of Traumatology and Orthopaedic Rehabilitation) since last year (2001). The clinical outcome has been found to be similar regardless of which interlocking nail system is being used. Management of distal third femoral fractures is one of the most serious and co problems over decades. The advent of interlocking device is revolutionary in solving problem and retrograde nailing is a promising technique [5]. But needs sophisticated instruments like C-arm and technical expertise. We are lacking in these modern equipment's and cannot provide all of our orthopaedic centers with $\mathrm{C}$-arm and expertise. In the recent past years, we have $\mathrm{C}$-arm in the National Traumatology and Orthopaedic Rehabilitation (NITOR) and by this time, sufficient expertise has grown up and we have done successfully some interlocking nailing in femoral shaft fractures. The purpose of this study is to evaluate a series of distal femoral shaft fractures treating by retrograde "SIGN" nail. If it is proved needful by this series it can be used as an alternative to other forms of distal shaft fractures management, $\mathrm{g}$ in distal third femoral shaft fractures are good.

\section{Methodology}

The prospective Study was conducted in the Department of Orthopaedic Surgery of Dhaka Medical College Hospital, Dhaka, Bangladesh to find out the common indications of Distal Third Femoral Shaft Fracture. Clinical examination and evaluation were done from January 2005 to December 2006. Other necessary investigations were done if clinically indicated and to prepare the patient for anesthesia. 15 cases were randomly selected for the study whose common indication of Distal Third Femoral Shaft Fracture. In most cases, injury was high energy trauma due to motor vehicle accident and there was a preponderance of fracture on the right side $(70 \%)$ than the left side $(30 \%)$. Thirty patients with distal third femoral fractures were treated with retrograde interlocking nailing. Twenty-six patients were male and four patients were female. The mean age was 40.5 years (range 20-70 years). Two patients have ipsilateral tibia fractures and fixed with interlocking "SIGN" nail. Most fractures were Winquist type I \& II and three fractures were type-Ill. Most fractures were high-energy injuries caused by road traffic accidents. Purposive sampling method was followed as per inclusion and exclusion criteria and 15 patients were selected. All patients reported after trauma and were resuscitated and fixed at earlier possible date. Maximum patients were operated upon within 2 weeks. The circulation and motor function of the extremity are documented and a careful search is made on the ipsilateral lower extremity injuries. When femoral shaft fractures are caused by high-energy accidents, the following roentgenograms are obtained: cervical spine films, chest X-ray. A/P view of pelvis and $\mathrm{A} / \mathrm{P}$ and lateral views of the knee. Surface traction is applied to tore length at the fracture site. Early application of surface traction is a critical it of initial fracture care; it reduces haemorrhage at the fracture site, reduces and maintains alignment of the extremity. Inclusion criteria were patients with distal third femoral shaft fractures were taken as samples, fresh injuries and less than three weeks old fractures were taken and closed fractures were included in this study. Exclusion criteria were open fractures, fractures without epiphyseal fusion, multiple fractures and pathological fractures. Statistical analysis of the results was obtained by using window-based computer software devised with Statistical Packages for Social Sciences (SPSS-22).

\section{RESULTS}

Total patient was 15. Right and left sided involvement was 11 and 4 respectively. Mean age was 40.5 years (range 20-70 years). Male -female ratio was $13: 2$. Out of 15,13 cases sustained high-energy trauma, of which 12 cases due to road traffic accident. Table I: shows that the age range of patients was 20-70 years (mean 40.5 years). Majority of the patients belonged to age group of 30-39 years (30\%). Regarding the mechanism of injury, table- $\mathrm{V}$ shows that the highest percentage $(86.67 \%)$ had sustained high-energy trauma while only $13.33 \%$ suffered low-energy trauma. Study shows that 18 patients were operated upon within 1-2 weeks of trauma. Figure III shows 7 fractures were 
locked statically and 5 fractures dynamically. Out of 15 , 3 fractures were dynamized. Among static nailing (7 cases). 2cases rated as excellent result, 4 cases rated as good. One case rated as fair and 0 case rated as poor result. Among dynamic nailing (5 cases), 2 cases rated as excellent, I case rated as good, 1 case rated as fair and poor. 1 case rated as excellent result, 1 case rated as good, 1 case rated as fair result and 0 rated as poor result.

Table-I: Demonstrated the distribution of study patients according to age $(n=15)$.

\begin{tabular}{|l|l|l|}
\hline Age group (years) & $\mathbf{n = 1 5}$ & $\mathbf{\%}$ \\
\hline 20 years to 29 years & 3 & 20.0 \\
\hline 30 years to 39 years & 5 & 33.33 \\
\hline 40 years to 49 years & 3 & 20.0 \\
\hline 50 years to 59 years & 3 & 20.0 \\
\hline 60 years to 70 years & 1 & 6.67 \\
\hline
\end{tabular}

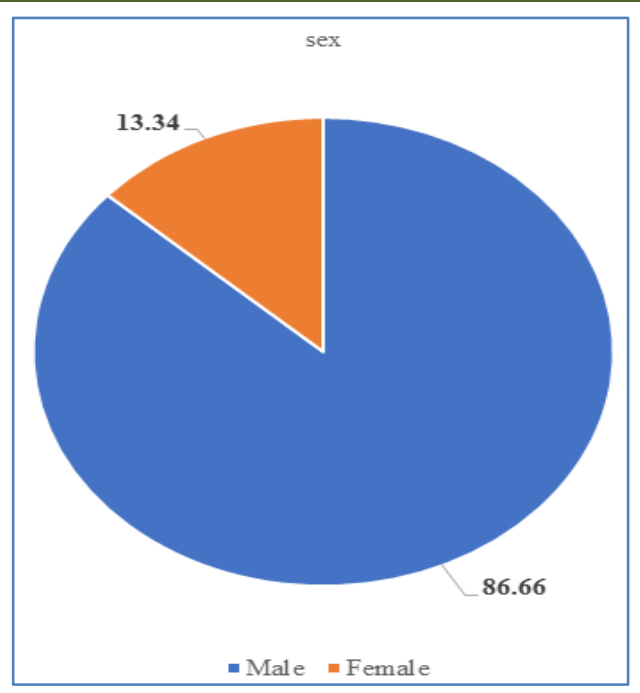

Fig-I: Demonstrated the distribution of study patients according to sex $(n=15)$

Table-II: Demonstrated the distribution of study patients according to Mechanism of injury $(\mathrm{n}=15)$

\begin{tabular}{|l|l|l|}
\hline Mechanism & $\mathbf{n = 1 5}$ & $\mathbf{\%}$ \\
\hline High-energy trauma & 13 & 86.67 \\
\hline Motor vehicle accident (bus, car) & 07 & 46.67 \\
\hline Motor cycle accident & 02 & 13.33 \\
\hline Auto-pedestrian accident & 03 & 20 \\
\hline Fall from height & 01 & 10 \\
\hline Low-energy trauma (Fall from bed, chair) & 02 & 13.33 \\
\hline
\end{tabular}

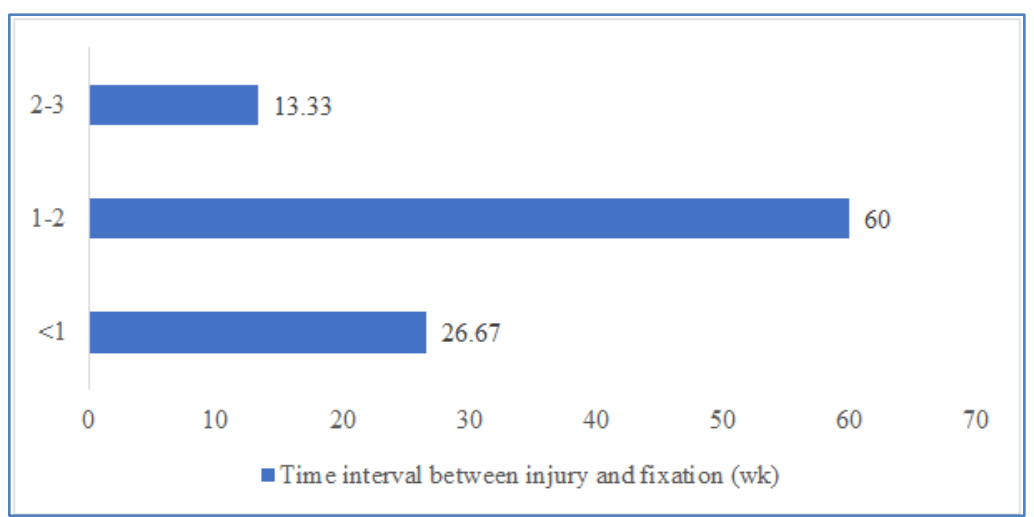

Fig-II: Demonstrated the distribution of study patients according to time interval between injury and fixation of fracture $(\mathbf{n}=15)$

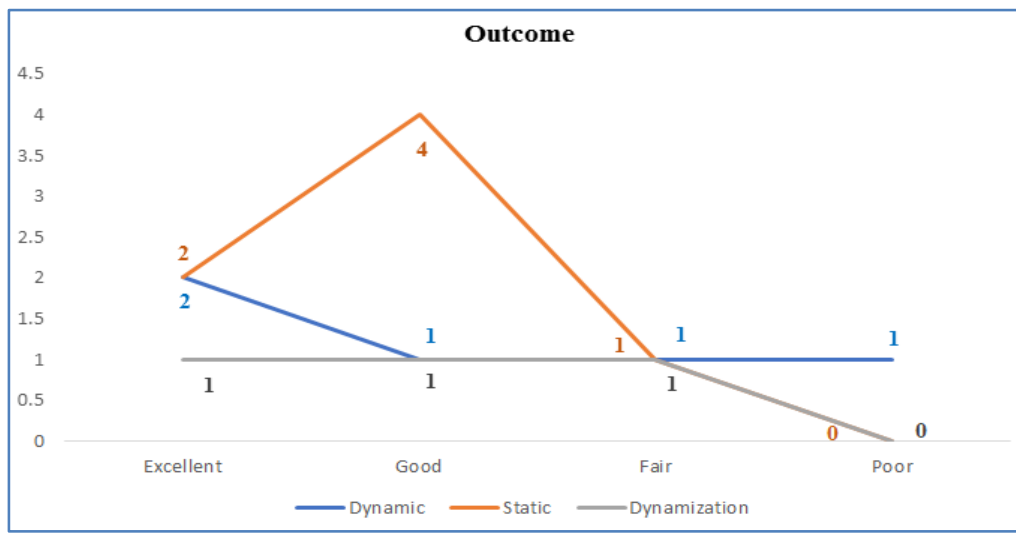

Fig-III: Demonstrated the distribution of study patients according to Outcome $(n=15)$ 


\section{DISCUSSION}

Fractures of the distal third of the femoral shaft remain in a therapeutic challenge. Historically, the majority of these fractures were treated conservatively. The muscle attachments of the distal femur produce characteristic fracture deformities; however, with improvements in surgical technique and implant technology, surgery is the treatmént of choice today [6]. The results of retrograde reamed interlocking nailing are distal third femoral fractures have been reported by numerous investigations [7-9]. Comparison among these studies are often difficult and wide variations frequently exist. Some of these discrepancies may be attributed to factors including patient selection, various methods of rehabilitation after injury, and differences in length of follow-up. Patients of these injuries or the presence of concomitant injuries, but with regard to age, sex, lifestyle and level of activity. In the present series, the age of the patients varied from minimum 20 years to maximum 70 years, mean age being 40.5 years. When compared to sex as male-female ratio, the incidence of series. Regarding the nature of trauma resulting in distal femoral shaft fractures; it was found that majority $(90 \%)$ occurred due to high-energy trauma. Out of 90 percent. $50 \% \%$ and $20 \%$ of fractures of distal femur occurred due to motor vehicle and auto-pedestrian accidents respectively. Only $10 \%$ of distal femoral fractures occurred due to low energy trauma as a result of domestic fall or other accidents. Interlocking intramedullary nailing is specially advantageous in comminuted fractures that has tendency towards rotational instability and shortening in the capacious area of distal third femoral shaft. In this series some comminuted fractures were treated with both statically and dynamically locked retrograde nails. The results of the present series were comparable with the reports of retrograde interlocking in distal third femoral fractures $[4,10]$.

This series had a few problems of malalignment as those reported. Varus and valgus angulations $(4.4 \%)$ were 5 degrees and 7 degrees respectively in the series some study did not find any malunion in their series. In this series 2 patients developed posterior 6 degrees valgus developed 7 degrees varus deformity. Some study did not find shortening in their series. In this study, shortening of femur developed in three patients of $1 \mathrm{~cm} .1 .5 \mathrm{~cm}$. and 2 $\mathrm{cm}$ [11-13]. Two were treated statically locked nail and one was treated dynamically locked nail and there were some degrees of comminution at the fracture site was dynamized. Two patients $(6.66 \%)$ developed superficial surgical wound infection: one at the knee and another at the thigh. The infection was treated by local wound dressing pus was sent for culture and sensitivity, which yielded growth of staphylococcus aureus in mostly resistant to crystalline penicillin, ampicillin, amoxicillin, cloxacillin, cotrimoxazole. Staphylococcus aureus was sensitive to ciprofloxacin, gentamycin and cephalexin. Culture of wound swab trom the yielded growth of mixed bacteria (Staphylococcus aureus, Escherichia coli, Pseudomona \& porteus), mostly resistant to crystalline penicillin, ampicillin amoxicillin, cloxacillin, cotrimoxazole. They were sensitive to ciprofloxacin, gentamycin and cephalexin. Both the patients responded well to antibiotics used according to the culture and sensitivity report and healed quickly. But some study did not find any infection in their series $[14,15]$. In this series, all cases had closed fractures and prophylactic antibiotic ( ${ }^{\text {rd }}$ generation cephalosporin) had been started preoperatively and continued postoperatively for 2 weeks. Postoperatively all patients were in close supervision. In this series, manipulation of two knees under G/A was done after 12 weeks due to stiffness. None of the cases had received physiotherapy adequately and came for follow-up after 12 weeks of discharge. One patient gained 120 degrees range of knee motion after manipulation. Rupture of quadriceps tendon occurred in another patient during manipulation and attempt was made for repair of the tendon in the next operation day. But the patient did not agree for operation and did not come for follow-up later on. The fracture was uniting in this patient. Manipulated knee in two patients due to knee stiffness in their series. Regarding range of knee motion, nine $(30 \%)$ patients had full range of motion, fourteen $(46.66 \%)$ patients had average range of knee motion 120 degrees, four $(13.33 \%)$ patients had average range of knee motion 110 degrees and in three (10\%) cases, range of knee motion was average 90 degrees. In the series of some study range of knee motion was average 117 degrees [13].

Ninety percent of the fractures were united in this series and were capable of fall weight bearing. The fracture union was defined as the period between injury and full weight bearing. Roentgenographically healed fracture was characterized by cortical bridging in tow views. Period of fracture healing was 14 to 28 weeks (average 18.27 weeks) in this series. Period of fracture healing was average 18.1 weeks in the series of [11], 22.4 weeks in the series. Nonunion occurred in $3(10 \%)$ patients. After one year, on bridging of the fracture-line was seen. In these patients there were distraction at the fracture site and comminution at the fracture ends. Two of the 3 fractures were statically locked and one was dynamically locked was dynamized but did not unite. These 3 cases of nonunion were referred to the parent units because of ending of my thesis period, where these patients were managed by curettage of fibrous tissue from the fracture site followed by bone grafting. Some study did not find any nonunion in their series $[13,14]$. Delayed union occurred in $6(20 \%)$ patients in this series. Four of the six fractures were dynamized and ultimately healed. Remaining two fractures healed without further intervention. In this series some of the patients complained mild to moderate type of pain over the thigh and ipsilateral knee after operation and 
Erfanul Huq siddiqui et al; Sch J App Med Sci, Apr, 2021; 9(4): 583-587

improved after union of bone. Three patients complained of constant pain at the fracture site and knee. In final follow-up, the satisfactory result (excellent and good) of this series was $76.66 \%$. Satisfactory result of some study was $90 \%$ [13]. In conclusion, retrograde reamed interlocking intramedullary nailing is useful in the treatment of the distal femoral shaft fractures. This study was done in thirty patients and the follow-up period was short (average 11.5 months). So, further prospective study, larger sample and longer period of follow-up are required to delineate the long term outcome of the knee joint function.

\section{CONCLUSION}

The management of distal femoral shaft fractures constitutes a therapeutic dilemma; the problems are primarily related to basic anatomic structure and biomechanical stresses of this region. Conservative method resulted in high rates of morbidity. So, open reduction and internal fixation is the treatment of choice in distal third femoral fracture. Distal femoral shaft fractures are included in this study and among the different methods of treatment, only open reduction with internal fixation by retrograde interlocking "SIGN" nailing was applied. The time subjective classification in this study showed 30 pert excellent, 46.66 percent good, 13.33 percent fair and 10 percent poor results. The presence of malalignment, knee stiffness and pain were poor prognostic sign. The results of the present study suggest that retrograde nailing in distal third of the femoral shaft fracture is one of the fracture managements in selected group of patients.

\section{REFERENCES}

1. Mathysen A. Du bandage platre et de son application dans le traitement des fractures. Grandmont-Donders; 1854.

2. Mays J, Neufeld AJ. Skeletal traction methods. Clinical Orthopaedics and Related Research (1976-2007). 1974 Jul 1;102:144-51.

3. Kuntscher G. The Detensor nail for fracture shaft of femur. Clin Ortho. 1968;75:143-50.
4. Leggon RE, Feldmann DD. Retrograde femoral nailing: a focus on the knee. The American journal of knee surgery. 2001 Jan 1;14(2):109-18.

5. Alien W, Piotrowsky G, Burnstein A, Frankel V. Biochemical principles of intramedullary fixation. Clin Orthop. 1968; 60:13-20.

6. Kenneth J, Koval MD. Supracondylar and Intramedullar Fractures of the Distal Femur. In: Alan M, Levine MD editor. Orthopaedic Knowledge Update Trauma. American Academy of Orthopaedic Surgeons. 1998; pp.137-43.

7. Koval KJ, Seligson D, Rosen H, Fee K. Distal femoral nonunion: treatment with a retrograde inserted locked intramedullary nail. J Orhtop Trauma. 1995; 9(4): 285-91.

8. Herscovici F Jr, Whiteman KW. Retrograde nailing of the femur using an intercondylar approach. Clin Orthop. 1996; (332): 98-104

9. Jangzing HM, Stockman B, Van Damme G, Rommens P, Broos PL. The retrograde intramedullary supracondylar nail; an alternative in the treatment of distal femoral fractures in the elderly. Arch. Orthop Trauma Surg. 1998; 118(12):92-5.

10. Derscovici D Jr, Whiteman KW. Retrograde nailing of the femur using an intercondylar approach. Clin Orthop. 1986; 332: 98-104.

11. Dicicco JD 3, Jenkins M, Ostrum RF. Retrograde nailing for subtrochanteric femur fractures. AM J Orthop. 2000 Sep; 29(9suppl); 4-8.

12. Handolin L, Pajarinen J, Tulikoura I. Injury to the deep femoral artery during proximal locking of a distal femoral nail-a report of 2 cases. Acta Orthopaedica Scandinavica. 2003 Jan 1;74(1):1113.

13. Leggon RE, Feldmann DD. Retrograde femoral nailing: a focus on the knee. The American journal of knee surgery. 2001 Jan 1;14(2):109-18.

14. Tzioupis C, Giannoudis PV. Prevalence of longbone non-unions. Injury. 2007 May 1;38:S3-9.

15. Tornetta III P, Tiburzi D. Reamed versus nonreamed anterograde femoral nailing. Journal of orthopaedic trauma. 2000 Jan 1;14(1):15-9.

16. Leggon RE, Feldmann DD. Retrograde femoral nailing: a focus on the knee. The American journal of knee surgery. 2001 Jan 1;14(2):109-18. 